\title{
IRIS study: a phase II study of the steroid sulfatase inhibitor Irosustat when added to an aromatase inhibitor in ER-positive breast cancer patients
}

\author{
Carlo Palmieri ${ }^{1,2,3}$ (D) Rob C. Stein ${ }^{4}$ Xinxue Liu $^{3}$ Emma Hudson ${ }^{3}$. \\ Hanna Nicholas $^{3}$ - Hironobu Sasano ${ }^{5}$ Fouzia Guestini $^{5}$ - Chris Holcombe ${ }^{6}$. \\ Sophie Barrett ${ }^{7} \cdot$ Laura Kenny $^{3} \cdot$ Sadie Reed $^{3} \cdot$ Adrian Lim $^{8} \cdot$ Larry Hayward $^{9}$. \\ Sacha Howell ${ }^{10} \cdot$ R. Charles Coombes ${ }^{3} \cdot$ On behalf of the IRIStrial participants
}

Received: 28 April 2017/Accepted: 1 June 2017/Published online: 13 June 2017

(C) The Author(s) 2017. This article is an open access publication

\begin{abstract}
Purpose Irosustat is a first-generation, orally active, irreversible steroid sulfatase inhibitor. We performed a multicentre, open label phase II trial of the addition of Irosustat to a first-line aromatase inhibitor (AI) in patients with advanced $\mathrm{BC}$ to evaluate the safety of the combination and to test the hypothesis that the addition of Irosustat to AI may further suppress estradiol levels and result in clinical benefit.

Experimental design Postmenopausal women with ERpositive locally advanced or metastatic breast cancer who had derived clinical benefit from a first-line AI and who subsequently progressed were enrolled. The first-line AI was continued and Irosustat (40 mg orally daily) added. The primary endpoint was clinical benefit rate (CBR).
\end{abstract}

Electronic supplementary material The online version of this article (doi:10.1007/s10549-017-4328-z) contains supplementary material, which is available to authorized users.

Carlo Palmieri

c.palmieri@liverpool.ac.uk

1 Department of Molecular and Clinical Cancer Medicine, University of Liverpool, Liverpool L69 3BX, UK

2 Academic Department of Medical Oncology, Clatterbridge Cancer Centre NHS Foundation Trust, Wirral CH63 4JY, UK

3 Department of Surgery and Cancer, Imperial College London, London W12 0NN, UK

4 NIHR University College London Hospitals Biomedical Research Centre, London NW1 2PG, UK

5 Department of Pathology, Tohoku University School of Medicine, Sendai, Japan

6 Breast Unit, The Linda McCartney Centre, Royal Liverpool University Hospital, Liverpool L7 8XP, UK
Secondary endpoints included safety, tolerability, and pharmacodynamic end points.

Results Twenty-seven women were recruited, four discontinued treatment without response assessment. Based on local reporting, the CBR was $18.5 \% \quad(95 \%$ CI $6.3-38.1 \%$ ) on an intent to treat basis, increasing to $21.7 \%$ (95\% CI $7.4-43.7 \%$ ) by per-protocol analysis. In those patients that achieved clinical benefit $(n=5)$, the median (interquartile range) duration was 9.4 months (8.1-11.3) months. The median progression-free survival time was 2.7 months (95\% CI 2.5-4.6) in both the ITT and perprotocol analyses. The most frequently reported grade $3 / 4$ toxicities were dry skin (28\%), nausea (13\%), fatigue $(13 \%)$, diarrhoea $(8 \%)$, headache $(7 \%)$, anorexia $(7 \%)$ and lethargy (7\%).

Conclusions The addition of Irosustat to aromatase inhibitor therapy resulted in clinical benefit with an acceptable safety profile. The study met its pre-defined success criterion by both local and central radiological assessments.

7 Beatson West of Scotland Cancer Centre, Glasgow G12 0YN, UK

8 Department of Radiology, Imperial College NHS Foundation Trust, London W8 6RF, UK

9 Edinburgh Cancer Centre, Western General Hospital, Edinburgh EH4 2XU, UK

10 Department of Medical Oncology, The University of Manchester, The Christie NHS Foundation Trust, Manchester M20 4BX, UK 
Keywords Breast cancer - Endocrine therapy - Sulfatase · Aromatase

\section{Introduction}

Inhibition of the activity of the transcription factor oestrogen receptor alpha (ER) represents a cornerstone strategy in the management of ER-positive $(\mathrm{ER}+)$ breast cancer (BC). Lowering of circulating estradiol levels through the inhibition of peripheral aromatase enzyme activity is one of the key endocrine manipulations used in the management of postmenopausal BC $[1,2]$. However, the second major pathway for oestrogen biosynthesis, steroid sulfatase (STS), is yet to be exploited therapeutically.

STS is responsible for the hydrolysis of steroid sulfates to their unconjugated, biologically active forms converting estrone sulphate (E1S) and DHEAS to estrone and DHEA, respectively. STS mRNA is expressed in the majority of $\mathrm{ER}+$ breast tumours and is inversely associated with survival [3, 4]. Expression of STS protein has been reported in $74 \%$ of breast cancers and its expression is significantly associated with larger tumour size, and with an increased risk of recurrence and poorer overall survival [5]. Conversely, the expression of oestrogen sulfotransferase (EST), which opposes the actions of STS, inversely correlates with tumour size, lymph node status and is significantly associated with a decreased risk of recurrence and improved overall survival [5]. The importance of DHEAS as a precursor for androstenediol was shown by its ability to stimulate the proliferation of breast cancer cells, which could be blocked with an anti-oestrogen or STS inhibitor, but not an AI [6]. Serum DHEAS levels have been shown to be significantly elevated in women progressing on an AI [7] suggesting that androstenediol production from DHEAS may be a mechanism of AI resistance. This is supported by data from a neoadjuvant study, which found an increase in STS following exposure to an aromatase inhibitor (AI) [8]. This could therefore represent a compensatory and adaptive response to the blockade of aromatase and the subsequent depletion of intratumoral oestrogen.

Irosustat (STX64) a tricyclic coumarin sulphamate is a first-generation irreversible inhibitor of STS [9]. Two phase I studies of Irosustat have been performed [10, 11]. In the first, 14 women who had progressed on two prior lines of endocrine therapy (ET) were treated with either 5 or $20 \mathrm{mg}$ doses of Irosustat [10]. STS activity as measured in peripheral blood lymphocytes (PBLs) and within the breast tumours was almost completely inhibited following treatment with Irosustat. As predicted by its mechanism of action, there was a significant suppression of serum estrone, estradiol, androstenediol and DHEA [10]. Four patients (all previously progressed on AI) had stable disease for 2.75-7 months. In the second study, performed following reformulation, the optimal biological dose (OBD) was found to be $40 \mathrm{mg}$ daily based on the reduction of STS activity in peripheral blood, changes in circulating steroidogenic hormones and the lack of grade 3 toxicity in the first 28 days [11]. No objective responses were seen, and the median time to progression for the $40 \mathrm{mg}$ group was 10.1 [(3.0-72.3) weeks]. Disease stabilisation was seen in three of thirteen patients at the $40 \mathrm{mg}$ dose, all of whom remained progression free for at least 24 weeks (range 27.1-72.3 weeks). An FDG-PET-scan sub-study carried out in six patients at the $40 \mathrm{mg}$ dose revealed that $50 \%$ of patients displayed significant median decreases in standardised uptake mean value (SUVmean), and hypermetabolic tissular volume (HT Volume) at Day 28 [11]. In both studies, Irosustat was well tolerated with no biochemical or haematologic toxicities $[10,11]$. Based on the safety profile of Irosustat from these initial phase I studies and the known side effect profile of AIs, no safety issues were expected with regard to combining Irosustat with an AI. Therefore, a phase II combination study was developed.

The IRIS study was designed to investigate the efficacy and tolerability of Irosustat in postmenopausal women who had progressed on a first-line aromatase inhibitor (AI) from which they had derived clinical benefit. This study aimed to test the hypothesis that the blockade of STS with Irosustat on the background of continued aromatase inhibition could result in clinical benefit. Safety and pharmacodynamic endpoints were also assessed.

\section{Materials and methods}

\section{Study design}

The IRIS study (ClinicalTrials.gov identifier NCT0178 5992) was a multicentre, open label phase II trial performed in nine academic medical centres in the United Kingdom (full list in supplementary information). Ethical approval was given by the NRES Committee LondonRiverside (an Independent Ethics Committee; reference 12/LO/0477), as well as being approved by the United Kingdom Medicines and Healthcare Products Regulatory Agency (EudraCT: 2011-005680-25). All patients provided written informed consent. 


\section{Eligibility}

Women were eligible if they were postmenopausal, with histologically confirmed ER-positive, HER-2 negative inoperable locally advanced or metastatic breast cancer. ER positivity was based on local laboratory assessment. Patients had to have developed progressive disease during first-line AI treatment for recurrent ER-positive breast cancer. Furthermore, patients had to have derived clinical benefit, defined as a documented objective response at any point or disease stabilisation (SD) for at least 6 months, from this first-line AI treatment. The disease had to be measurable by CT/MRI scan according to RECIST v1.1. (Full inclusion and exclusion criteria in supplementary information).

\section{Trial treatment}

Irosustat was given orally at a dose of $40 \mathrm{mg}$ daily in addition to the first-line AI, which was continued beyond progression. No other therapy was given in the intervening time between progression on the AI and commencement of Irosustat. Combined therapy was continued until disease progression, death, the development of unacceptable toxicities or the withdrawal of consent.

\section{Trial assessments}

Clinical assessments and toxicity reporting were performed every month (28 days) for the first 6 months and 3 monthly thereafter until disease progression, occurrence of unacceptable toxicities or withdrawal from treatment. A safety visit was performed 7 days after Irosustat was discontinued. Tumour response was evaluated every 3 months according to RECIST version 1.1. Adverse events (AEs) were graded according to National Cancer Institute Common Terminology Criteria (version 4.3), with relationship to study medication recorded, and coded using the Medical Dictionary for Regulatory Activities (MedDRA version 14.0). Formalinfixed paraffin-embedded (FFPE) samples were requested of the primary tumour and any recurrence or metastatic site that had been biopsied prior to study entry. Blood samples were collected and processed at baseline, every month and on progression (see supplementary information).

\section{Study endpoints}

The primary endpoint was clinical benefit rate which was defined as the proportion of patients with either complete response (CR) or partial response (PR) at any scheduled tumour assessment, or stable disease (SD) for at least 6 months. Secondary endpoints included progression-free survival (PFS), defined as time from study enrolment to first evidence of PD or death due to any cause, duration of clinical benefit as defined by the number of days from start of study drug to the first evidence of progressive disease (PD) or death due to any cause; objective response rate defined by the proportion of CR and PR. Safety and tolerability as assessed by the collection of adverse events (AE) according to the Common Terminology Criteria for Adverse Events (NCI-CTCAE $\mathrm{v}$ 4.03) and to measure alterations in circulating steroid hormones and correlate these measures with clinical outcome. Exploratory translational endpoints included the assessment of the expression of steroidogenic enzymes, i.e. STS, aromatase, EST, 17ßHSD1 and 17ßHSD2. Central review of all study imaging was undertaken by an independent radiologist.

\section{Steroidogenic hormone profiling}

Steroidogenic Hormone profiling was carried out by a central laboratory, Quest Diagnostics (Nichols Institute, San Juan Capistrano, CA, USA). Androstenedione, oestrone sulfate (E1S), dehydroepiandrosterone sulphate (DHEAS), dehydroepiandrosterone (DHEA), androstenediol and testosterone were quantitated using a TSQ Quantum Ultra (Thermo Fisher; San Jose, CA) triple quadrupole tandem mass spectrometer. Estrone and estradiol were detected and quantitated in negative ionisation mode using a triple quadrupole tandem mass spectrometer with APCI source (TSQ Quantum Ultra, Thermo Fisher; San Jose, CA). Further detailed methodology is provided in the supplementary materials and methods.

\section{Immunohistochemistry staining}

Immunohistochemistry was performed for the expression of four enzymes involved in oestrogen metabolism (aromatase, steroid sulfatase, oestrogen sulfotransferase, 17beta-Hydroxysteroid dehydrogenase type 1 and type 2); detailed methodology is described in supplementary materials and methods.

Immunostained slides were independently evaluated by two of the authors (FG, HS) who are experienced in scoring the four biomarkers concerned, both were blinded to patients' clinical outcomes. Marker's expression was evaluated by assigning scores for the approximate percentage of immunopositive cells (proportion score) and for staining intensity, which were added together. The range of proportion scores for aromatase was $0-3$ as follows: $0 \leq 1 \%, \quad 1=1-25 \%, \quad 2=26-50 \% \quad$ and $3 \geq 50 \%$ immunopositive cells (Sasano et al. 2009). The range of proportion score for STS, EST and 17BHSD was 0-2 as follows: $0=$ no stained tumour cells, $1=1-50 \%$, $2 \geq 50 \%$ immunopositive cells (5). Relative staining 
intensity of immunopositive cells was classified as $0=$ no immunoreactivity, $\quad 1$ = weak, $\quad 2$ = moderate and $3=$ strong immunoreactivity.

\section{Statistical analysis}

The study used a Simon's minimax two-stage design to provide $80 \%$ power with a one-sided type I error of 0.05 to declare the treatment effective assuming a maximum unacceptable CBR (p0) of $5 \%$ and a minimum acceptable CBR (p1) of 20\%. In the first stage, 13 patients were to be evaluated, and if at least one patient achieved clinical benefit, another 14 patients would be enrolled. If the clinical benefit was seen in 4 out of 27 patients overall, the treatment would be declared effective. For PFS, patients were censored at the time of the last follow-up if they had withdrawn or been lost to follow-up before progression or death. PFS was estimated using the Kaplan-Meier method. All 27 patients enrolled in the study and who received at least one dose of drug, formed the Intent to treat population, while the per-protocol group included all patients except three patients who withdraw themselves and one who was withdrawn by the local principal investigator prior to the first tumour assessment at 3 months.

\section{Results}

Between February 2013 and March 201428 patients were consented. One patient was found to be ineligible prior to starting treatment. All 27 patients enrolled in the study received at least one dose of drug, and formed the ITT population. Four patients withdrew from the study before the first tumour assessment (Fig. 1). The baseline clinicopathological details of patients are provided in Table 1. Prior to recruitment, all 27 patients were receiving an AI as first-line therapy with a median (IQR) duration of treatment of 21.1 (13.3-37.6) months; $27 \%$ of patients had received one course of chemotherapy for advanced disease.

Drug compliance was monitored using a patient diary. Of the 27 patients in the study, drug compliance data were available for 26 patients as one patient mislaid her drug diary. The compliance with both AI treatment and Irosustat during the study was very good with median rates of $100 \%$ (range $90.5-100 \%$ ) and $100 \%$ (range $87.0-100 \%$ ), respectively.

\section{Efficacy}

For the 27 patients recruited, the median duration of treatment was 2.8 months (range 1.5-17.4 months). Based on the local study sites' tumour assessment, there were no objective responses. At the interim analysis when the primary outcome for the first 13 patients was available, we observed three patients with clinical benefit (three stable diseases for at least 6 months) and the trial moved to the second stage, where another two patients with clinical benefit were observed. In summary, five patients had stable disease for at least 6 months giving a clinical benefit rate (CBR) of $18.5 \%$ (95\% CI 6.3-38.1\%) on an intent to treat basis (Table 2). In a perprotocol analysis which excluded the four patients who withdrew before the first tumour assessment, the CBR was $21.7 \%$ (95\% CI 7.4-43.7\%).

Central review was undertaken for all patients where at least one radiological assessment had been undertaken (i.e. the per-protocol group) which confirmed that there were no objective responses (Table 2). As a result of the central review, four patients derived clinical benefit, giving a CBR of $14.8 \%$ (95\% CI $4.2-33.7 \%$ ) based on intent to treat analysis and $17.4 \%$ (95\% CI 5.0-38.8\%) in the per-protocol analysis.

The median (IQR) duration of clinical benefit in the local ITT analysis of 26 patients was 9.4 months (8.1-11.3); the precise date of starting study drug was unknown for one patient. PFS based on local review was 2.7 months (95\% CI 2.5-4.6) in both the ITT and the perprotocol analysis (Fig. 2).

\section{Adverse events}

All twenty-seven patients experienced treatment-emergent adverse events, $91 \%$ of which were grade 1 or 2 (Table 3). The most common were dry skin (77\%), nausea (48\%) and fatigue $(40 \%)$. Grade 2 ECG abnormalities (QT prolongation) were reported in one patient, which were considered unrelated to study drug. Three patients were discontinued due to AEs; these were urinary tract infection, possible renal toxicity (not reported as AE) and dry skin.

Nine serious adverse events occurred in 6 patients (22\%). None were considered definitely related to study drug; one was considered probably related (nausea and vomiting) and another possibly related (nausea and vomiting). All other SAEs were considered as either unlikely to be related (sepsis, urinary tract infection, breast pain) or not related (cellulitis secondary to an animal bite, symptoms of morphine toxicity, pneumonia). There was one death due to bronchopneumonia during the study that was unrelated to the study medication.

\section{Pharmacodynamic data}

At study entry and at all subsequent monthly time points, the circulating levels of estradiol and estrone were below the threshold of detection in all patients $(2$ and $10 \mathrm{ng} / \mathrm{dl}$, 
Fig. 1 CONSORT trial diagram. ITT intent to treat, $P I$ principal investigator

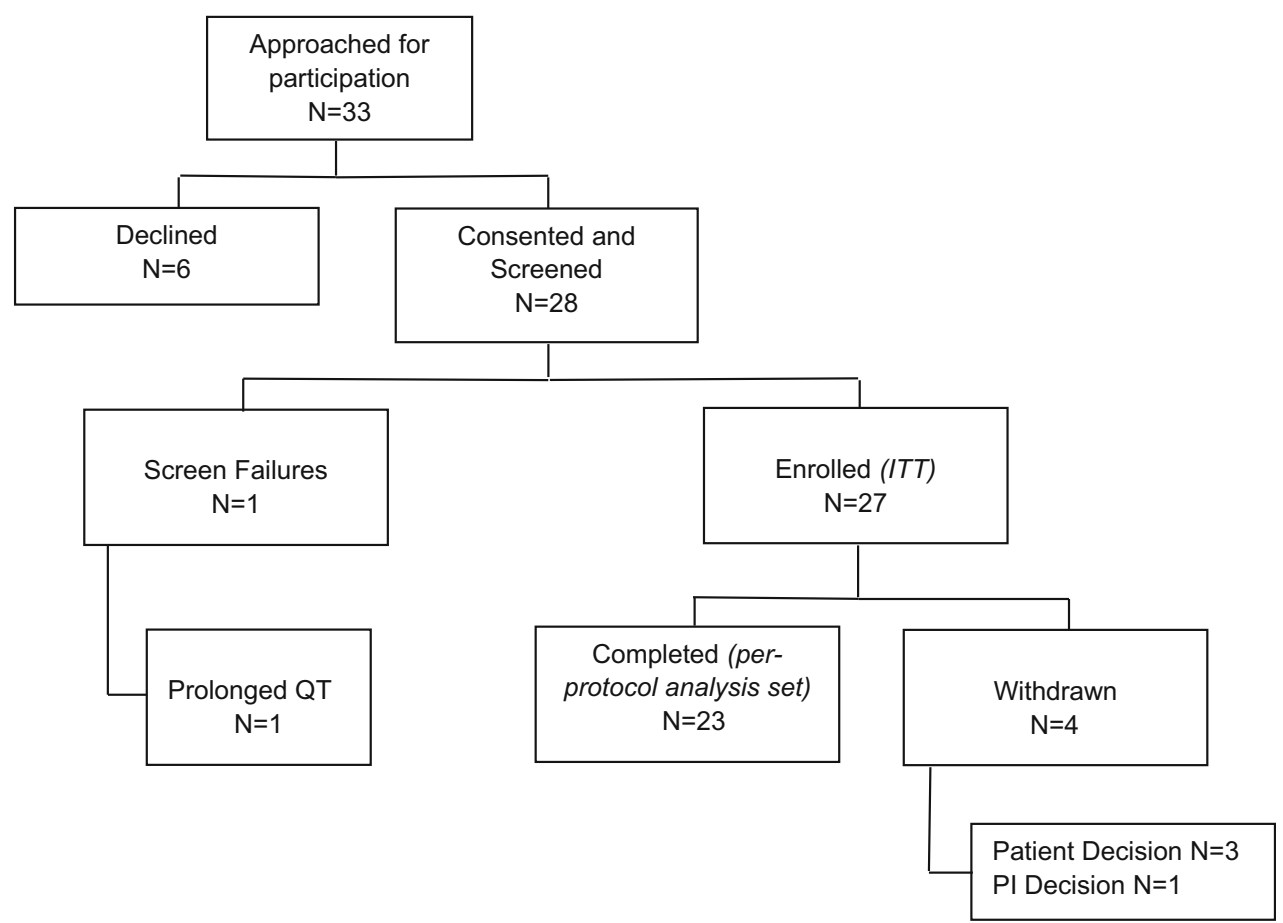

androstenedione (Adione), much of it is rapidly sulphated to estrone sulfate (E1S), by the enzyme oestrogen sulfotransferase (EST) [12]. E1S is known to have a plasma concentration 10-20 times higher than those of the unconjugated oestrogens estrone and estradiol, as well as a longer half-life in the plasma than the unconjugated oestrogens and therefore acts as a reservoir for active estrogens [13]. E1S uptake into malignant breast tissue is facilitated by organic anion transporter polypeptide B (OATP) [14]. Secondly, androgens derived from the adrenal cortex are known to have oestrogenic effects [15]. Thirdly, STS, the enzyme responsible for the hydrolysis of steroid sulphates to their unconjugated biologically active forms, is overexpressed in breast cancer and is known to be associated with a worse outcome $[4,5]$. Fourthly, STS is upregulated following exposure to an aromatase inhibitor (AI) which is a putative mechanism of resistance to AIs [8]. Fifthly, the additive use of oestrogen synthesis inhibition can result in clinical benefit as seen when an $\mathrm{AI}$ is added to ovarian suppression in premenopausal women with breast cancer [16]. We therefore hypothesised that the addition of an STS inhibitor to an AI at disease progression would result in a lowering of peripheral and intratumoral estrone and DHEA, reversal of resistance and clinical benefit.

The principal findings relate to effect on disease status, adverse events and effects on steroid hormones. Firstly, regarding the anti-tumour effects of Irosustat, the study met its primary endpoint of showing evidence of clinical benefit in a second-line setting based on both local and central reviews, with a median PFS of 2.7 months. Although there 
Table 1 Clinicopathological details

\begin{tabular}{|c|c|c|}
\hline & ITT $N=27$ & Per-protocol analysis $N=23$ \\
\hline Age (years) & $63.7(10.5)$ & $63.3(9.9)$ \\
\hline BMI $\left(\mathrm{kg} / \mathrm{m}^{2}\right)$ & $28.1(6.3)$ & $28.2(6.6)$ \\
\hline Duration of the AI treatment at enrolment (month) ${ }^{\mathrm{a}}$ & $21.1(13.3-37.6)$ & $21.1(10.3-35.7)$ \\
\hline Time between primary diagnosis to metastatic diagnosis (month) ${ }^{\mathrm{a}}$ & $60.7(2.0-116.1)[n=23]$ & $60.7(2.0-105.4)[n=19]$ \\
\hline \multicolumn{3}{|l|}{ Ethnicity } \\
\hline White & $24(88.9 \%)$ & $21(91.3 \%)$ \\
\hline Asian & $1(3.7 \%)$ & $1(4.4 \%)$ \\
\hline Black & $1(3.7 \%)$ & $0(0 \%)$ \\
\hline Chinese & $1(3.7 \%)$ & $1(4.4 \%)$ \\
\hline \multicolumn{3}{|l|}{ No. of sites of disease } \\
\hline 1 & $7(25.9 \%)$ & $6(26.1 \%)$ \\
\hline 2 & $9(33.3 \%)$ & $8(34.8 \%)$ \\
\hline 3 & $7(25.9 \%)$ & $7(30.4 \%)$ \\
\hline 4 & $1(3.7 \%)$ & $0(0 \%)$ \\
\hline 5 & $2(7.4 \%)$ & $1(4.4 \%)$ \\
\hline Missing & $1(3.7 \%)$ & $1(4.4 \%)$ \\
\hline \multicolumn{3}{|l|}{ Visceral disease } \\
\hline No & $6(22.2 \%)$ & $6(26.1 \%)$ \\
\hline Yes & $20(74.1 \%)$ & $16(69.6 \%)$ \\
\hline Missing & $1(3.7 \%)$ & $1(4.4 \%)$ \\
\hline \multicolumn{3}{|l|}{ ER status ${ }^{b}$} \\
\hline Positive & $27(100 \%)$ & $23(100 \%)$ \\
\hline Negative & $0(0 \%)$ & $0(0 \%)$ \\
\hline \multicolumn{3}{|l|}{ PgR status ${ }^{\mathrm{b}}$} \\
\hline Positive & $20(74.1 \%)$ & $16(69.6 \%)$ \\
\hline Negative & $4(14.8 \%)$ & $4(17.4 \%)$ \\
\hline Unknown & $3(11.1 \%)$ & $3(13.0 \%)$ \\
\hline \multicolumn{3}{|l|}{ HER2 status ${ }^{\mathrm{b}}$} \\
\hline 0 & $10(37.0 \%)$ & $10(43.5 \%)$ \\
\hline $1+$ & $10(37.0 \%)$ & $7(30.4 \%)$ \\
\hline $2+$ & $3(11.1 \%)$ & $2(8.7 \%)$ \\
\hline Amplified & $0(0 \%)$ & $0(0 \%)$ \\
\hline $3+^{c}$ & $1(3.7 \%)$ & $1(4.4 \%)$ \\
\hline Not done & $3(11.1 \%)$ & $3(13.0 \%)$ \\
\hline \multicolumn{3}{|l|}{ Treatment history: chemotherapy } \\
\hline No & $10(37.0 \%)$ & $9(39.1 \%)$ \\
\hline Yes & $17(63.0 \%)$ & $14(60.9 \%)$ \\
\hline Neoadjuvant & $2(9.1 \%)$ & $1(5.9 \%)$ \\
\hline Adjuvant & $14(63.6 \%)$ & $11(64.7 \%)$ \\
\hline Advanced 1st line & $6(27.3 \%)$ & $5(29.4 \%)$ \\
\hline \multicolumn{3}{|l|}{ Treatment history: radiotherapy } \\
\hline No & $13(48.1 \%)$ & $11(47.8 \%)$ \\
\hline Yes & $14(51.9 \%)$ & $12(52.2 \%)$ \\
\hline Adjuvant & $19(63.3 \%)$ & $18(66.7 \%)$ \\
\hline Palliative & $11(36.7 \%)$ & $9(33.3 \%)$ \\
\hline \multicolumn{3}{|l|}{ Treatment history: endocrine therapy } \\
\hline No & $0(0 \%)$ & $0(0 \%)$ \\
\hline Yes & $27(100.0 \%)$ & $23(100.0 \%)$ \\
\hline
\end{tabular}


Table 1 continued

\begin{tabular}{lcc}
\hline & ITT $N=27$ & Per-protocol analysis $N=23$ \\
\hline Neoadjuvant & & $1(2.5 \%)$ \\
$\quad$ Anastrozole & $1(2.1 \%)$ & \\
Adjuvant & & $1(2.5 \%)$ \\
$\quad$ Exemestane & $1(2.1 \%)$ & $1(2.5 \%)$ \\
Letrozole & $1(2.1 \%)$ & $3(7.5 \%)$ \\
Anastrozole & $4(8.5 \%)$ & $13(32.5 \%)$ \\
Tamoxifen & $16(34.0 \%)$ & $3(7.5 \%)$ \\
Advanced 1st line & & $14(35.0 \%)$ \\
Exemestane & $3(6.4 \%)$ & $4(10.0 \%)$ \\
$\quad$ Letrozole & $17(36.2 \%)$ & $4(8.5 \%)$ \\
$\quad$ Anastrozole & 4 & \\
\hline
\end{tabular}

ITT intent to treat

Data presented are mean (SD) for continuous variables and frequency (percentage) for categorical variables

${ }^{a}$ Data presented are median (inter quartile range)

b Based on diagnostic biopsy and primary tumour sample

c HER2 results: $3+$ on diagnostic biopsy and $1+$ on resected tumour

were no objective responses, a number of patients experienced disease stabilisation for at least 6 months. It is known that the objective response and clinical benefit rates of second-line endocrine treatment are limited. For example, the objective response rate to second-line endocrine therapy in large phase III studies with exemestane or highdose fulvestrant has been reported to be in the range of 0.4-6.3\%, while median PFS in these studies ranged from 2.8 to 3.8 months [17-19]. Therefore, Irosustat as a single agent added onto the background of an AI has evidence of clinical activity and, with the caveats relating to cross trial comparison and their limitations, does show efficacy which is comparable to both exemestane and high-dose fulvestrant in this setting. Future studies need to explore the clinical activity of dual aromatase and STS blockade as first-line metastatic treatment in a patients likely to be endocrine sensitive, as well selecting for STS expression.

The suppression of circulating estradiol is a key therapeutic strategy in the management of ER-positive breast cancer, and all the women who entered this study had estrone and estradiol levels below the level of detection as the result of AI treatment. However, steroidogenic hormones derived from the adrenal gland have been implicated in AI resistance. DHEAS is the most abundant steroid in the human circulation and has been shown to be significantly elevated in women whose disease failed to respond to an $\mathrm{AI}$ in the metastatic setting [7]. DHEA has been shown to stimulate the proliferation of the oestrogendependent MCF-7 human breast cancer cell line, as well as a derived oestrogen-independent variant, and to transactivate ER in both lines [20]. In vitro DHEAS has been shown to transactivate both ER and the AR in a dose-dependent manner, with the DHEAS induced AR transactivity abolished by Irosustat [21]. DHEA can itself be converted to androstenediol by $17 \beta-\mathrm{HSD} 1$, as well as to androstenedione via $3 \beta-H S D 1$ and $17 \beta-H S D 2$. Androstenediol is known to have potent oestrogenic effects [15], and can stimulate the growth of hormone-dependent breast cancer cells both in vitro and in vivo [22, 23]. Androstenedione has been demonstrated to induce recruitment of ER and SRC3 to gene promoters and drive ER transcription [24] and $\mathrm{AI}$ resistance in vitro [25]. Androstenedione was significantly elevated in women on a second-line AI for metastatic breast cancer as compared to women being treated in the adjuvant setting [26]. Furthermore, androstenedione can be converted to $5 \mathrm{a}$-androstane- $3 \mathrm{~b}$, 17b-diol (3bAdiol) which is itself estrogenic, and can induce growth in breast cancer cells [27]. This compound has also been implicated in endocrine resistance [28]. Clinically, previous studies have also shown that AIs do not affect the levels of androstenediol [29], while serum DHEAS was significantly higher in women who progressed on an AI [7]. Steroids with potent estrogenic properties may provide an escape mechanism for growth within a low estradiol milieu.

Steroidogenic hormone measurements within the current study revealed treatment with Irosustat resulted in significant and predicable rise at 3 months in estrone sulphate and DHEAS, and decrease in DHEA:DHEAS as well as significant reduction in androstenedione at 3 months. These pharmacodynamics data confirm that Irosustat had effected sulfatase inhibition in our patients. Expression of STS and 
Table 2 Efficacy analysis on the basis of local and central assessment

\begin{tabular}{lll}
\hline & ITT $[n=27]$ & Per protocol $[n=23]$ \\
\hline $\begin{array}{lll}\text { Local assessment } \\
\text { Clinical benefit rate }\end{array}$ & $18.5 \%(6.3-38.1 \%)$ & $21.7 \%(7.4-43.7 \%)$ \\
Response at 6 month scan & \\
Complete response & 0 & 0 \\
Partial response & 0 & 0 \\
Stable disease & 5 & 5 \\
Progressive disease & 16 & 16 \\
Dead & 1 & 1 \\
Withdrawal & 5 & 1 \\
Central assessment & & \\
Clinical benefit rate & $14.8 \%(4.2-33.7 \%)$ & $17.4 \%(5.0-38.8 \%)$ \\
Response at 6 month scan & \\
Complete response & 0 & 0 \\
Partial response & 0 & 0 \\
Stable disease & 4 & 4 \\
Progressive disease & 15 & 15 \\
Dead & 1 & 1 \\
Withdrawal & 7 & 3 \\
\hline
\end{tabular}

ITT intent to treat; Prior to 3 month scan: three patients decided to withdraw; one patient withdrawn by local investigator. These four patients had no scan and were excluded in the per-protocol analysis; Between 3 and 6 month scan: one patient withdrawn

${ }^{a}$ At month 3: two patients PD reclassified as SD by central review and thus treated as withdrawals

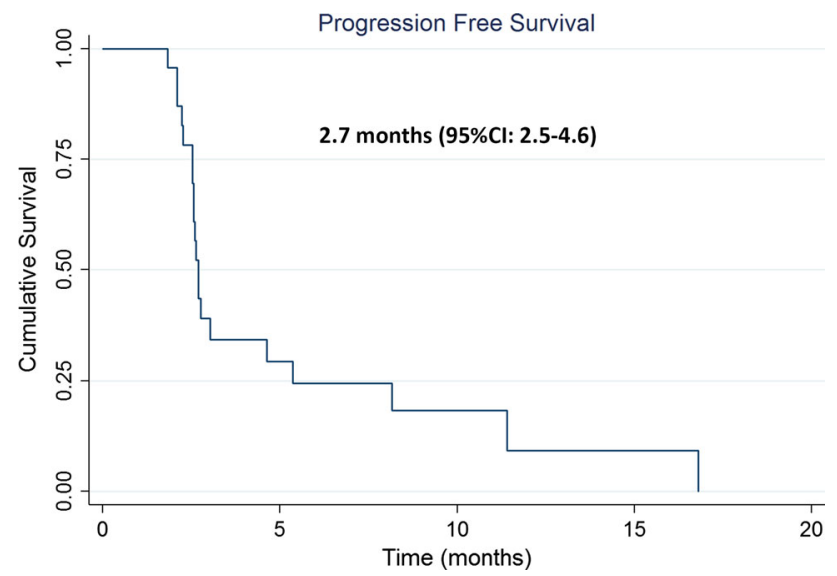

Fig. 2 Progression-free survival as assessed by the investigators in the intention-to-treat population

other enzymes involved in oestrogen biosynthesis were assessed predominately in primary breast cancer samples. Given the small numbers, no formal assessment can be made of the possible use of STS as a biomarker of response. However, moderate to strong expression of STS was observed in only 37 and $25 \%$ of the primary and
Table 3 Treatment-emergent adverse events regardless of relationship to study drugs

\begin{tabular}{|c|c|c|c|}
\hline Adverse event & Grade $1-2$ & Grade $3-4$ & Grade 5 \\
\hline Dry skin & 21 & 1 & \\
\hline Nausea & 13 & 2 & \\
\hline Fatigue & 11 & 2 & \\
\hline Diarrhoea & 8 & 0 & \\
\hline Decreased appetite & 5 & 0 & \\
\hline Headache & 5 & 0 & \\
\hline Lethargy & 5 & 0 & \\
\hline Vomiting & 4 & 0 & \\
\hline Rash & 3 & 1 & \\
\hline Cough & 3 & 0 & \\
\hline Dizziness & 3 & 1 & \\
\hline Arthralgia & 3 & 0 & \\
\hline Insomnia & 3 & 0 & \\
\hline Sepsis & 0 & 1 & \\
\hline Haemoglobin & 0 & 1 & \\
\hline Urinary tract infection & 0 & 1 & \\
\hline Breast ulceration & 0 & 1 & \\
\hline Gamma GT increase & 0 & 2 & \\
\hline Blurred vision & 0 & 1 & \\
\hline Bone pain & 0 & 1 & \\
\hline Pleurodesis & 0 & 1 & \\
\hline Pneumonia & 0 & 0 & 1 \\
\hline
\end{tabular}

Grades 1 and 2 with an incidence of $10 \%$ of study population and all grade $3-5$ toxicities

recurrent cases, and the preponderance of tumours with low STS expression clearly may have impacted on the efficacy data. Furthermore, the primary tissue was mainly used and this may not reflect the biology of a tumour which has progressed following a prolonged period of oestrogen deprivation. Ideally, in future studies of Irosustat a metastatic biopsy should be mandated prior to study entry [30], and prospectively enrich for a STS high population of tumours.

Irosustat was well tolerated with most adverse effects being grades 1 and 2 , and the most common being dry skin, nausea and fatigue. Dry skin is an expected side effect being reported in both phase I studies [10, 11]. This is expected given that hereditary deficiency of STS results in dry, scaly skin as a result of abnormal corneocyte retention and thickening of the stratum corneum [31].

There are several limitations to our study. Primary tumours were utilised for the IHC assessment since metastatic biopsies from the time of study entry were not routinely collected [30], particularly given the evidence that STS is upregulated following exposure to an AI [8]. Since the study was initiated, the development of ESR1 


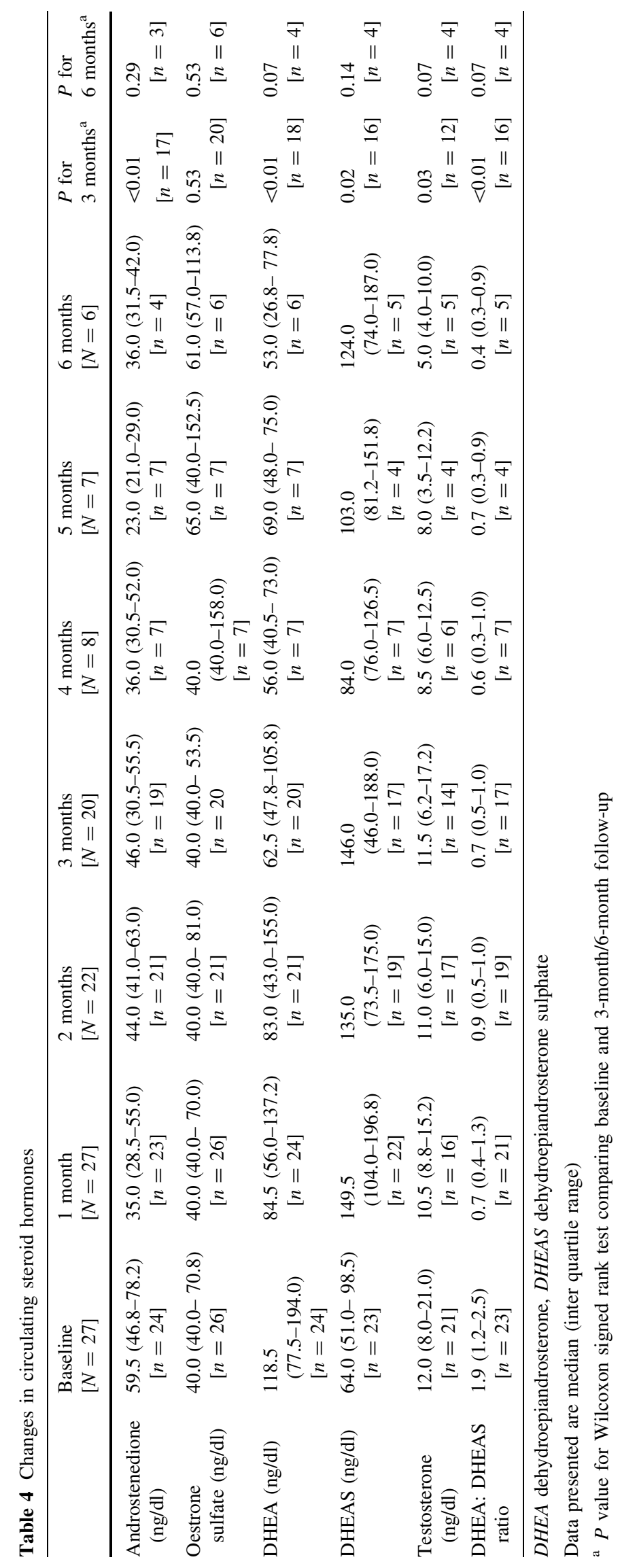


mutations as a resistance mechanism to AIs has been described. As all patients who entered the study had progressed on an AI, it would be expected that $20-30 \%$ would harbour ESR1 mutations [32-34]. The possible effect of ESR1 mutations with regard to the efficacy of Irosustat is unknown. The study strengths are that we undertook local and central review of radiology, with the study meeting its primary endpoint by both assessments.

More recently, it has been demonstrated that the addition of either an mTOR inhibitor or a CDK4/6 inhibitor to an AI can significantly improve the outcomes of women with ER-positive metastatic breast cancer [17, 35]. Given the clinical activity of these targeted therapies is dependent on the dual targeting of ER with endocrine therapy, the development of a more effective ET backbone may lead to enhance efficacy of these biological therapies. Therefore, any future clinical development will need to attempt to address the question of whether an ET backbone which comprises it and an AI is more clinically effective than an AI alone when combined with targeted therapies such an mTOR or CDK4/6 inhibitors.

In summary, this proof of concept study provides evidence for the first time that combining both an AI and a STS inhibitor can have clinical activity and that the combination is safe and well tolerated. Future studies should explore the clinical activity of Irosustat at an early line of treatment in a populations enriched for STS expression.

Acknowledgements We thank the women who took part in this study; the doctors, nurses and support staff at the following local sites: Royal Liverpool University Hospital (4 patients); University College London Hospital (3 patients); Beatson West of Scotland Cancer Centre (1 patient); Imperial College NHS Foundation Trust, London (10 patients); Western General Hospital (4 patients); The Christie NHS Foundation Trust (5 patients). We also thank the independent members of the trial steering committee (Prof David Miles (Chair); Dr Richard Simcock; Dr Laura Assersoh; Mr Olorunsola Agbaje) and the independent data monitoring committee (Alastair Thompson (Chair); Dr Mark. Beresford; Dr Louise Choo). IRIS was an NIHR Clinical Research Network portfolio trial and we acknowledge the help of the local research networks that supported recruitment at UK sites. We are grateful to Dr Nigel J. Clarke, Quest Diagnostics for support with the steroidogenic hormone profiling and aromatase inhibitor drug levels. We are particularly grateful to Adrienne Morgan, patient representative and member of Independent Cancer Patients' Voice for her input, guidance and help with patient information sheet and for her participation in the study steering committee meetings.

Financial support The study was supported by a feasibility study award from Cancer Research UK (grant number C20208/A13392), and recruitment was supported via the National Institute for Health Research (NIHR), as well as an educational grant from IPSEN. The funders had no role in study design, data collection and analysis, decision to publish or preparation of the manuscript. RCS was supported by the National Institute for Health Research University College London Hospitals Biomedical Research Centre.

\section{Compliance with ethical standards}

Conflicts of interest The authors declare no potential conflicts of interest.

Open Access This article is distributed under the terms of the Creative Commons Attribution 4.0 International License (http://crea tivecommons.org/licenses/by/4.0/), which permits unrestricted use, distribution, and reproduction in any medium, provided you give appropriate credit to the original author(s) and the source, provide a link to the Creative Commons license, and indicate if changes were made.

\section{References}

1. Santen RJ, Brodie H, Simpson ER, Siiteri PK, Brodie A (2009) History of aromatase: saga of an important biological mediator and therapeutic target. Endocr Rev 30:343-375

2. Palmieri C, Patten DK, Januszewski A, Zucchini G, Howell SJ (2014) Breast cancer: current and future endocrine therapies. Mol Cell Endocrinol 382:695-723

3. Utsumi T, Yoshimura N, Maruta M, Takeuchi S, Ando J, Mizoguchi Y et al (2000) Correlation of cyclin D1 mRNA levels with clinico-pathological parameters and clinical outcome in human breast carcinomas. Int J Cancer 89:39-43

4. Utsumi T, Yoshimura N, Maruta M, Takeuchi S, Ando J, Maeda $\mathrm{K}$ et al (1999) Significance of steroid sulfatase expression in human breast cancer. Breast Cancer 25:298-300

5. Suzuki T, Nakata T, Miki Y, Kaneko C, Moriya T, Ishida T et al (2003) Estrogen sulfotransferase and steroid sulfatase in human breast carcinoma. Can Res 63:2762-2770

6. Billich A, Nussbaumer P, Lehr P (2000) Stimulation of MCF-7 breast cancer cell proliferation by estrone sulfate and dehydroepiandrosterone sulfate: inhibition by novel non-steroidal steroid sulfatase inhibitors. J Steroid Biochem Mol Biol 73:225-235

7. Morris KT, Toth-Fejel S, Schmidt J, Fletcher WS, Pommier RF (2001) High dehydroepiandrosterone-sulfate predicts breast cancer progression during new aromatase inhibitor therapy and stimulates breast cancer cell growth in tissue culture: a renewed role for adrenalectomy. Surgery 130:947-953

8. Chanplakorn N, Chanplakorn P, Suzuki T, Ono K, Chan MS, Miki Y et al (2010) Increased estrogen sulfatase (STS) and 17beta-hydroxysteroid dehydrogenase type 1(17beta-HSD1) following neoadjuvant aromatase inhibitor therapy in breast cancer patients. Breast Cancer Res Treat 120:639-648

9. Malini B, Purohit A, Ganeshapillai D, Woo LW, Potter BV, Reed MJ (2000) Inhibition of steroid sulphatase activity by tricyclic coumarin sulphamates. J Steroid Biochem Mol Biol 75:253-258

10. Stanway SJ, Purohit A, Woo LW, Sufi S, Vigushin D, Ward R et al (2006) Phase I study of STX 64 (667 Coumate) in breast cancer patients: the first study of a steroid sulfatase inhibitor. Clin Cancer Res 12:1585-1592

11. Coombes RC, Cardoso F, Isambert N, Lesimple T, Soulié P, Peraire $C$ et al (2013) A phase I dose escalation study to determine the optimal biological dose of irosustat, an oral steroid sulfatase inhibitor, in postmenopausal women with estrogen receptor-positive breast cancer. Breast Cancer Res Treat 140:73-82

12. Hobkirk R (1993) Steroid sulfation current concepts. Trends Endocrinol Metab 4:69-74 
13. Ruder H, Loriaux L, Lipsett M (1972) Estrone sulfate: production rate and metabolism in man. J Clin Investig 51:1020-1033

14. Pizzagalli F, Varga Z, Huber RD, Folkers G, Meier PJ, St-Pierre MV (2003) Identification of steroid sulfate transport processes in the human mammary gland. J Clin Endocrinol Metab 88:3902-3912

15. Adams J, Garcia M, Rochefort H (1981) Estrogenic effects of physiological concentrations of 5-androstene- 3 beta, 17 beta-diol and its metabolism in MCF7 human breast cancer cells. Cancer Res 41:4720-4726

16. Stein RC, Dowsett M, Hedley A, Gazet JC, Ford HT, Coombes RC (1990) The clinical and endocrine effects of 4-hydroxyandrostenedione alone and in combination with goserelin in premenopausal women with advanced breast cancer. $\mathrm{Br} \mathrm{J}$ Cancer 62:679-683

17. Baselga J, Campone M, Piccart M, Burris HA 3rd, Rugo HS, Sahmoud $\mathrm{T}$ et al (2012) Everolimus in postmenopausal hormonereceptor-positive advanced breast cancer. NEJM 366:520-529

18. Johnston SR, Kilburn LS, Ellis P, Dodwell D, Cameron D et al (2013) Fulvestrant plus anastrozole or placebo versus exemestane alone after progression on non-steroidal aromatase inhibitors in postmenopausal patients with hormone-receptor-positive locally advanced or metastatic breast cancer (SoFEA): a composite, multicentre, phase 3 randomised trial. Lancet Oncol 14:989-998

19. Turner NC, Ro J, André F, Loi S, Verma S, Iwata H et al (2015) Palbociclib in hormone-receptor-positive advanced breast cancer. N Engl J Med 373:209-219

20. Maggiolini M, Donze O, Jeannin E, Ando S, Picard D (1999) Adrenal androgens stimulate the proliferation of breast cancer cells as direct activators of estrogen receptor alpha. Cancer Res 59:4864-4869

21. Bjerregaard-Olesen C, Ghisari M, Kjeldsen LS, Wielsøe M, Bonefeld-Jørgensen EC (2016) Estrone sulfate and dehydroepiandrosterone sulfate: transactivation of the estrogen and androgen receptor. Steroids 105:50-58

22. Poulin R, Labrie F (1986) Stimulation of cell proliferation and estrogenic response by adrenal C19-delta 5-steroids in the ZR-751 human breast cancer cell line. Cancer Res 46:4933-4937

23. Dauvois S, Labrie F (1989) Androstenedione and androst-5-ene-3 beta,17 beta-diol stimulate DMBA-induced rat mammary tumors-role of aromatase. Breast Cancer Res Treat 13:61-69

24. Shin I, Miller T, Arteaga CL (2006) ErbB receptor signaling and therapeutic resistance to aromatase inhibitors. Clin Cancer Res $12: 1008 \mathrm{~s}-1012 \mathrm{~s}$
25. O'Hara J, Vareslija D, McBryan J, Bane F, Tibbitts P, Byrne C et al (2012) AIB $1: e R \alpha$ transcriptional activity is selectively enhanced in aromatase inhibitor-resistant breast cancer cells. Clin Cancer Res 18:3305-3315

26. Elliott KM, Dent J, Stanczyk FZ, Woodley L, Coombes RC, Purohit A et al (2014) Effects of aromatase inhibitors and body mass index on steroid hormone levels in women with early and advanced breast cancer. Br J Surg 101:939-948

27. Sikora MJ, Strumba V, Lipmann ME, Johnson MD, Rae JM (2012) Mechanisms of estrogen-independent breast cancer growth driven by low estrogen concentrations are unique versus complete estrogen deprivation. Breast Cancer Res Treat 134:1027-1039

28. Sikora MJ, Cordero KE, Larios JM, Johnson MD, Lippman ME, Rae JM (2009) The androgen metabolite 5alpha-androstane-3beta,17beta-diol (3betaAdiol) induces breast cancer growth via estrogen receptor: implications for aromatase inhibitor resistance. Breast Cancer Res Treat 115:289-296

29. Stanway SJ, Palmieri C, Stanczyk FZ, Folkerd EJ, Dowsett M, Ward R et al (2011) Effect of tamoxifen or anastrozole on steroid sulfatase activity and serum androgen concentrations in postmenopausal women with breast cancer. Anticancer Res 31:1367-1372

30. Sharma A, Crook T, Thompson A, Palmieri C (2010) Why biopsying metastatic breast cancer should be routine. Nat Rev Clin Oncol 7:72-74

31. Marinković-Ilsen A, Koppe JG, Jöbsis AC, de Groot WP (1978) Enzymatic basis of typical X-linked ichthyosis. Lancet 2:1097

32. Fuqua SA, Wiltschke C, Zhang QX, Borg A, Castles CG, Friedrichs WE, Hopp T, Hilsenbeck S, Mohsin S, O'Connell P, Allred DC (2000) A hypersensitive estrogen receptor-alpha mutation in premalignant breast lesions. Cancer Res 60:4026-4029

33. Fuqua SA (2001) The role of estrogen receptors in breast cancer metastasis. J Mammary Gland Biol Neoplasia 6:407-417

34. Wang P, Bahreini A, Gyanchandani R, Lucas PC, Hartmaier RJ, Watters RJ et al (2016) Sensitive detection of mono- and polyclonal ESR1 mutations in primary tumors, metastatic lesions, and cell-free DNA of breast cancer patients. Clin Cancer Res 22:1130-1137

35. Hortobagyi GN, Stemmer SM, Burris HA, Yap YS, Sonke GS, Paluch-Shimon S et al (2016) Ribociclib as first-line therapy for HR-positive, advanced breast cancer. $N$ Engl J Med $375: 1738-1748$ 